\title{
Ocho años en bioética
}

Luis Moreno-Exebio ${ }^{1}$

Resumen: Este trabajo presenta la experiencia que significó el paso por el Programa Internacional de Formación en Ética de la investigación biomédica y psicosocial del Centro Interdisciplinario de Estudios en Bioética de la Universidad de Chile (20032004) y algunas reflexiones respecto de la integración de lo aprendido en mi posterior labor en comités de ética y programas académicos de ética de investigación. Asimismo, en la elaboración de regulaciones que permitan una mejor evaluación ética y científica.

Palabras clave: formación bioética, comités de ética, Centro Interdisciplinario de Estudios en Bioética, investigación con seres humanos

\section{Eight years of bioethics}

Abstract: This article presents the experience acquired by participating in the international ethics of biomedical and psychosocial research training program of the Interdisciplinary Center for Studies on Bioethics of the University of Chile (20032004) and some reflections about the integration of knowledge acquired in my posterior work at scientific ethical review committees and ethics of research academic programs. Furthermore, in the elaboration of regulations for improving scientific and ethical review.

Key Words: bioethics training, ethics of research committees, Interdisciplinary Center for Studies on Bioethics, research involving human beings

\section{Oito anos em bioética}

Resumo: Este trabalho apresenta a experiência que significou a passagem pelo Programa Internacional de Formação em Ética da Pesquisa Biomédica e Psicossocial do Centro Interdisciplinar de Estudos em Bioética da Universidade do Chile (2003-2004) e algumas reflexóes a respeito da integração do que foi aprendido em meu posterior labor em comitês de ética e programas acadêmicos de ética em pesquisa; assim como na elaboração de regulamentaçóes que permitam uma melhor avaliação ética e científica.

Palavras-chave: formaçáo bioética, comitês de ética, Centro Interdisciplinar de Estudos em Bioética, pesquisa com seres humanos

\footnotetext{
${ }^{1}$ Centro Nacional de Control de Calidad, Instituto Nacional de Salud, Perú. Fogarty NIH Research Grant \#D43 TW 06056. Año de egreso: 2004

Correspondencia: lemoreno70@hotmail.com
} 


\section{Introducción}

En setiembre de 2002 recibí un insólito documento: "Resolución Jefatural No 311-2002-J-OPD/ INS". En los considerandos de dicho documento se indicaba: "Se conforma el Sub-Comité de Ética del Instituto Nacional de Salud". Mi sorpresa fue grande cuando vi mi nombre dentro de la relación de miembros del comité, pero esta sorpresa inicial se fue convirtiendo en preocupación cuando adjuntos se me entregaban extensos documentos correspondientes al Reglamento y Procedimiento de Evaluación de Proyectos de Investigación Biomédica con Seres Humanos del Sub-Comité.

Comencé asistiendo a las sesiones, primero por curiosidad, porque nunca había estado en un comité de este tipo, y luego con el afán de aprender de los miembros más experimentados. Con el pasar del tiempo me fueron asignando protocolos o propuestas de investigación con seres humanos de mayor complejidad, que generaron en mí la necesidad de buscar una mayor preparación para afrontar este difícil reto.

A finales de 2002 llegó a mis manos una convocatoria para participar en un Programa Internacional de Formación en Ética de la Investigación en Seres Humanos en la Universidad de Chile; me presenté y en abril de 2003 estaba en Chile iniciando mis estudios formales en dicho Programa.

Las razones para postular al Programa fueron fundamentalmente porque tenía entre sus objetivos, por una parte, ofrecer a calificados profesionales de América Latina una formación multidisciplinaria en ética de la investigación biomédica y psicosocial, y, por otra, reforzar la capacidad de los países latinoamericanos para abordar los aspectos éticos y sociales de la investigación científica en el contexto de la investigación internacional. Ambos propósitos se enlazaban perfectamente con mi búsqueda de capacitación.

\section{Estructura del Programa}

El Programa tenía en ese momento una duración de doce meses; una formación que en sus inicios se dividía en ocho meses de actividades lectivas y prácticas en la Universidad de Chile, y durante los siguientes cuatro meses los participantes éramos asignados, de acuerdo con nuestros intereses, a una de las universidades latinoamericanas que participaba del Programa, para desarrollar diferentes actividades: participación en comités de ética, actividades de consultoría, académicas, entre otras. En mi caso, esta segunda etapa la realicé en el Instituto Tecnológico de Santo Domingo, República Dominicana.

Las actividades académicas curriculares incluían sesiones lectivas divididas en cinco áreas temáticas: Perspectivas en Bioética, Bioética y Aspectos Sociales, Destrezas y Herramientas, Estudios de Caso y Trabajo de Campo, e Investigación y Bioética. También había talleres prácticos y la preparación de trabajos individuales y de grupo para ser discutidos en clase.

El primer escollo que debí superar en mi formación fue el curso "Perspectivas Históricas y Filosóficas en Bioética", que incluía la lectura y el análisis de muchas corrientes filosóficas que sirvieron de base y sustento para el desarrollo de la bioética; sin embargo, durante mis estudios de pre y de posgrado no había llevado curso alguno de filosofía y la lectura de autores como Kant, Aristóteles, Apel, Otto, Engelhardt, Jonas, MacIntyre, entre otros, me resultaba bastante ardua.

Debo agradecer aquí a un excelente amigo y también filósofo, Agustín Estévez, quien me ayudó a entender estos textos y a comprender muchos aspectos de la disciplina bioética.

Otra novedad, antes que escollo, fue el curso "Métodos de Investigación", en el que pude conocer procedimientos relacionados con las ciencias sociales, como por ejemplo encuestas, estudios de caso, entrevistas en profundidad, observación, entrevistas grupales, entre otros, que, para alguien proveniente del área de la Farmacia y Bioquími$\mathrm{ca}$, en la que predominan los estudios analíticos experimentales, fue un completo descubrimiento, el cual, luego de varios ańos, he sabido aquilatar, especialmente en mi labor como miembro de comités de ética. 


\section{Participantes del Programa}

En la primera versión del Programa tuve la suerte de compartir con profesionales de diversas disciplinas y países - Bioquímica (España), Filosofía (Argentina), Obstetricia, Enfermería y Sociología (Chile), Derecho (México), Química y Farmacia (Perú) y Medicina (Ecuador)—, compañeros con los cuales hemos tenido la dicha de encontrarnos posteriormente en actividades relacionadas con la bioética y de estrechar vínculos de amistad hasta el presente.

Durante el programa nos convertimos en un grupo transdisciplinar, como escribe Eduardo Rodríguez(1). Aquí la bioética se vuelve transdisciplinar, ya que busca una convergencia transcendiendo y permeando a los demás. Los participantes pasan de ser médicos, filósofos o abogados, a ser bioeticistas médicos, filósofos o abogados. Y esto no es mero cambio de lugar de términos, sino un posicionamiento cualitativamente diferente que exige una nueva construcción disciplinaria.

\section{Actividades realizadas en Chile}

Aparte de las actividades lectivas, talleres y seminarios, tuvimos la oportunidad de asistir en calidad de observadores a las sesiones del Comité Ético Científico del Servicio de Salud Metropolitano Sur de Santiago, lo cual fue muy valioso por el nivel de argumentación y la metodología de trabajo de dicho Comité.

Mención aparte merece la propuesta conjunta: "Conformación de un comité de ética en los países latinoamericanos y del Caribe", presentada por los alumnos del Programa en el Congreso de Bioética 2003 de la Sociedad Chilena de Bioética. De acuerdo con el Dr. Fernando Lolas, director del Programa, el discurso bioético es integrador, transdisciplinar y dialógico, lo que permite mediar o "tender puentes" entre racionalidades, personas, instituciones y disciplinas(2). Todo esto fue lo que pusimos en práctica para llegar, luego de largas jornadas de debate, al producto final.

\section{Actividades realizadas en República Domini- cana}

Las actividades en República Dominicana, en los primeros meses de 2004, estuvieron marcadas por cambios en la Comisión Nacional de Bioética, la cual fue reestructurada a raíz de un estudio controversial, motivo de un estudio de caso, para evaluar los principios bioéticos y la conducción responsable de la investigación(3).

Por otro lado, revisamos y planteamos sugerencias a las "Guías Operacionales para Comités de Ética en Investigación Biomédica en República Dominicana”, en coordinación con la representación en ese país de la Organización Panamericana de la Salud; asimismo, revisamos los reglamentos internos de los comités de ética del Hospital General Plaza de la Salud y del Instituto Dermatológico y Cirugía de la Piel Dr. Humberto Bogaert Díaz.

Finalmente, complementamos nuestra estancia con el dictado de clases, sobre aspectos referidos a la bioética, a alumnos de pre y posgrado del Instituto Tecnológico de Santo Domingo.

Al final de esta permanencia entendimos los diversos matices que adopta la bioética en América Latina, a pesar de que compartimos los mismos problemas sociales e institucionales; la manera como se abordan estos problemas desde la perspectiva bioética es muy diferente entre nuestros países.

\section{Actividades realizadas con posterioridad a la terminación del Programa}

Al término del Programa, en abril de 2004, me reintegro al Instituto Nacional de Salud (INS) a trabajar en el Comité de Ética y en el área de Ensayos Clínicos, donde me uno al grupo que venía elaborando la propuesta de reglamentación sobre Ensayos Clínicos. Participo activamente en la preparación de los artículos referidos a los postulados éticos y la protección de los sujetos de investigación. 
Además, realizo inspecciones a centros de investigación, como parte del equipo multidisciplinario encargado de esta tarea, asisto a reuniones y talleres con los principales actores de los ensayos clínicos y, finalmente, en julio de 2006, con la publicación del "Reglamento de Ensayos Clínicos en el Perú", a través del Decreto Supremo D.S. 017-2006-SA(4), se hace realidad el esfuerzo del Instituto Nacional de Salud por tener una norma actualizada en este tema. Una descripción del proceso de formulación del Reglamento puede revisarse en Monografía de Acta Bioethica No3(5).

Lamentablemente, al poco tiempo de entrar en vigencia el Reglamento de Ensayos Clínicos y por razones cuestionables(6), en marzo del 2007 se forma una Comisión Técnica para revisar el D.S. 017-2006-SA y, mediante RM No 164225-2007, se presenta un proyecto modificatorio del Reglamento, el cual es aprobado y publicado mediante D.S. No 006-2007-SA el 08 junio de 2007, Modificatoria del Reglamento de Ensayos Clínicos(7). Actualmente, tanto el D.S. 0172006-SA como su modificatoria D.S. No 0062007-SA son las normativas vigentes en el Perú sobre ensayos clínicos.

\section{Comités de ética en el Perú}

El Reglamento de Ensayos Clínicos en el Perú(4), en su capítulo VII, hace referencia a los comités de ética, denominándolos "comités institucionales de ética en investigación (CIEI)", instancia de la institución de investigación constituida por profesionales de diversas disciplinas y miembros de la comunidad, encargada de velar por la protección de los derechos, seguridad y bienestar de los sujetos de investigación, a través, entre otras cosas, de la revisión y aprobación/opinión favorable del protocolo del estudio, la capacidad del (de los) investigador (es) y lo adecuado de las instalaciones, métodos y material que se usarán al obtener y documentar el consentimiento informado de los sujetos en investigación.

Al regresar al Comité de Ética del INS, mi participación fue más activa; se promovió la capacitación de todos los miembros; se actualizó su regla- mento y manual de procedimientos, recogiendo la experiencia de los comités ético-científicos del Servicio de Salud Metropolitano Sur de Santiago y del Comité de Ética de la Asociación Dominicana Pro Bienestar de la Familia -PROFAMILIA-, en los realicé una pasantía. Se promovió la formación de la Red Nacional de Comités de Ética en Investigación en el Perú, entre otras actividades. En 2007 y entre 2009 y 2010 tuve el honor de ser su presidente.

En 2004, por invitación del Instituto de Ética de la Facultad de Medicina de la Universidad Nacional Mayor de San Marcos, formé parte de su primer comité de ética, experiencia enriquecedora que me permitió conocer los problemas que se presentaban en la aplicación del nuevo reglamento aprobado, el nivel de conocimientos sobre ética y bioética que exhibían los alumnos de la Facultad de Medicina y sus dificultades para la presentación de los protocolos.

Este año 2012 he regresado a formar parte de un comité de ética, el de la Universidad Cayetano Heredia que, establecido en 1992, constituye uno de los más antiguos en el país, siendo además esta universidad la principal institución que realiza investigación en el Perú.

Creo que la principal tarea de un CEI es la protección de los sujetos de investigación; sin embargo, sin apoyo institucional, sin recursos y sin un marco regulatorio adecuado es poco lo que se puede hacer para cumplir esta delicada tarea a cabalidad. En el Perú, la regulación específica sobre comités de ética es una tarea todavía pendiente.

\section{Universidad de Miami}

La Iniciativa Panamericana de Bioética (PABIPan American Bioethics Initiative) es un programa de la Universidad de Miami financiado por el Centro Internacional Fogarty de los Institutos Nacionales de Salud de Estados Unidos.

El principal objetivo de esta actividad es promover la integridad de la investigación clínica y la conducta responsable de la investigación, fomen- 
tando el desarrollo de programas de educación continua en ética de la investigación, aprovechando la plataforma virtual del Programa de Educación en Ética de la Investigación, conocido como CITI por sus siglas en inglés (Collaborative Institutional Training Initiative)(8).

PABI intenta ser una herramienta para la colaboración participativa en la protección de sujetos humanos y la integridad científica entre los países de América Latina, el Caribe y los Estados Unidos de América. Para tal fin, PABI ha convocado a autoridades del sector de la salud, decisores políticos, miembros de comités de ética de investigación y representantes de la industria farmacéutica de la región a participar como becarios e instructores del Programa.

En este contexto, participé como instructor de PABI desde 2008 hasta 2011, siendo un miembro activo de la industria farmacéutica. Este enfoque constituye una innovación en cuanto al público objetivo de capacitación en ética y busca integrar en un mismo esfuerzo a los actores principales de la investigación clínica: los patrocinadores.

Con el apoyo de PABI se ha formado un grupo de trabajo local, denominado IPEBI (Instituto Peruano de Educación en Bioética e Investigación), que incluye a miembros de comités de ética, investigadores, industria farmacéutica, academia y organizaciones de investigación por contrato (CROs), con la finalidad de promover la capacitación en todos los estamentos del proceso de investigación y darle sostenibilidad a esta inicia- tiva. Se han adaptado 11 módulos del Programa CITI a la normatividad y contexto peruano; se preparó un nuevo módulo de capacitación sobre Investigación Clínica en el Perú y se desarrollaron seminarios, cursos y talleres, creando un espacio de reflexión en el cual se discute el contenido educativo que pueda servir para cubrir las necesidades del país.

\section{Conclusión}

Estos ocho años, luego de egresado del Programa, han sido un continuo aprender. Transitar por el camino de la bioética no ha sido fácil; muchas veces he estado sometido a los vaivenes de la política, a la presión de grupos de interés y a desencuentros; sin embargo, la experiencia ha sido por demás fructífera. He aprendido mucho de la bioética, del diálogo y de la comunicación entre personas; también acerca de la formulación, fundamentación y aplicación de principios morales en ambientes plurales y diversos.

Debo agradecer la formación brindada en el Programa y reconocer que continuamente realiza esfuerzos para integrar diferentes perspectivas y proyecciones, como fue la reunión realizada en septiembre de 2011, en el Instituto Nacional de Salud del Perú, donde tuvimos la oportunidad de encontrarnos con los egresados del Programa desde su versión inicial hasta la fecha, permitiendo la interacción entre diferentes instituciones e iniciativas relacionadas con la bioética y la ética de investigación de América Latina. 


\section{Referencias}

1. Rodríguez E. Temas para una Bioética Latinoamericana. Acta Bioethica 2009; 15(1): 87-93.

2. Lolas F. Bioética y Medicina. Santiago de Chile: Editorial Biblioteca Americana; 2002.

3. Rodríguez E, Moreno L. Los principios éticos y la conducción responsable de la investigación. En: Lolas F, Quezada A, Rodríguez E. (eds.) Investigación en Salud. Dimensión Ética. Santiago de Chile: CIEB Universidad de Chile; 2006.

4. Aprueban el Reglamento de Ensayos Clínicos en el Perú D.S. No 017-2006-SA. Diario Oficial El Peruano. Lima: 29 de julio de 2006.

5. Moreno L, Fuentes D, Rodríguez J. Reglamento de ensayos clínicos en Perú y prioridades de investigación. En: Lolas F. (editor) Dimensiones éticas de las regulaciones en salud. Monografías de Acta Bioethica No 3. Santiago de Chile: CIEB Universidad de Chile, Programa de Bioética OPS/ OMS; 2009.

6. Fuentes D, Minaya M. ¿Cuánto puede avanzar la ciencia y la tecnología médica en un período de seis meses en el Perú? Revista Brasileira de Bioética 2009; 5(1-4): 34-47.

7. Modifican el Reglamento de Ensayos Clínicos en el Perú D.S. No 006-2007-SA. Diario Oficial El Peruano. Lima: 8 de junio de 2007.

8. Litewka S, Goodman K, Braunschweiger P. El Programa CITI: Una alternativa para la capacitación en ética de la Investigación en América Latina. Acta Bioethica 2008; 14(1): 54-60.

Recibido: 6 de febrero de 2012

Aceptado: 27 de marzo de 2012 